\title{
Towards an Objective Quality Evaluation Framework for Haptic Data Reduction
}

\author{
Rahul Chaudhari ${ }^{* 1}$, Eckehard Steinbach ${ }^{\dagger 1}$, and Sandra Hirche ${ }^{\ddagger 2}$ \\ ${ }^{1}$ Institute for Media Technology, Technische Universität München, Germany \\ ${ }^{2}$ Institute for Automatic Control Engineering, Technische Universität München, Germany
}

\begin{abstract}
High packet rates in telepresence and teleaction systems pose grave challenges to teleoperation over existing communication infrastructure like the Internet. To counter these issues, efficient perceptually motivated packet-rate reduction schemes have been developed. These schemes are conventionally evaluated for perceived quality via subjective user tests. Such tests are time-consuming, expensive and require precise control of experimental conditions. Computer modeling of telepresence sessions can, on the other hand, bring repeatability, ease of observation, definite control over system parameters and task description and fairness of comparison. In this paper, we present first steps towards a methodology and a framework to model and simulate a networked haptic interaction and evaluate it objectively for the quality of experience. Towards this purpose, we model the human control action and haptic perception process in teleoperation. Our results show that simulations of these models for a range of data reduction scheme parameters produce quality estimates whose trend is comparable to carefully performed subjective user tests.
\end{abstract}

Index Terms: H.1.2 [User/Machine Systems]: haptics-haptic compression, quality of experience

\section{INTRODUCTION}

Over the past few decades, telepresence systems have progressed in a manner that tries to comprehensively transport and aid human control capabilities by reining in all relevant modalities - audition, vision and haptics. The acquisition, communication and display of audio and video are traditionally well researched upon, but as we shift focus towards haptics, we face more design complexities along with proportionately powerful capacities. The intricacies of involving haptics stem, for the most part, from the bidirectional interaction between man and machine, so that not only can he perceive the haptic feedback offered - similar to other modalities - but also physically act upon an environment to alter it. It is clear that it is of utmost importance to ensure a human-centric treatment to design and analysis of haptic systems from a perceptual perspective.

Quality evaluation in the context of such systems is a vast topic in general in that a number of factors ranging from control architectures employed, data communication policies and schemes, interplay between different modalities to design of human system interfaces (haptic devices) engaged for the purpose can populate the picture $[3,13,14]$. In our work however, we will primarily concentrate on the appraisal of quality relevant to schemes employed

*e-mail: rahul.chaudhari@tum.de

†e-mail: eckehard.steinbach@tum.de

†e-mail: hirche@1sr.ei.tum.de

IEEE World Haptics Conference 2011

21-24 June, Istanbul, Turkey

978-1-4577-0298-3/11/\$26.00 @2011 IEEE for the communication of haptic data and the artifacts arising out of their peculiarities. More specifically, compression schemes for haptic data, e.g. [7], that enable teleoperation over large distances without compromising system stability shall be inquired for humancentric perceptual quality.

Traditionally, media compression schemes are evaluated primarily through subjective user tests. These tests are time-consuming and expensive. This has led to the development of a number of perceptual models and methodologies for objective quality evaluation (OQE) in the audio and video fields $[11,18]$, something that haptics research currently lacks. Computer modeling and simulation of telepresence sessions with appropriate haptic perception models should be developed to rectify this situation. Such a computer model would simulate a dedicated and consistent human who gives honest opinions about the system under test, in effect removing inter-subject variability of subjective tests.

Unlike audio and video, the bidirectional nature of haptic interaction necessitates that human action be included in our analyses. Ideally, the human model should include the active behavior of the human (central nervous system and the neuromuscular system of the arm) and the haptic perceptual mechanism. By building, simulating and iteratively refining a signal-based objective quality evaluation framework that employs these models, we aim to be able to progress towards compression quality inference results previously only achievable by diligently designed psychophysical experiments.

In the past, a number of researchers have carried out quality evaluation of haptic feedback systems for different applications and purposes. Some early approaches to objective quality evaluation include [8] where the influence of packet loss and time delay on the displayed impedance is evaluated in a mathematical framework consisting of a perceptual model of the human. In [5], the authors performed experiments to gather subjective data for various criteria like fatigue, perceived rendering quality and degree of immersion, etc. in a haptic interaction. Membership functions were defined for each of these criteria and the user data was mapped to quality-of-experience results using fuzzy rules. In [1], Basdogan et al. conducted studies to evaluate the role of haptic feedback in human-human and human-machine haptic interactions. Based on measurements of specific response variables and questionnaires, conclusions were drawn regarding the influence of haptic feedback on task performance and a sense of togetherness with other participants. In [17], Sagardia et al. provide a framework of testing scenarios and measures that help objectively evaluate the quality of haptic rendering algorithms by comparing the response of the algorithms with the expected analytical one. Similar to [17], we take a signal-based approach to the evaluation of haptic feedback, fundamentally different from the previously mentioned approaches in $[8,1,5]$. But we include the human element of haptic interaction in our analyses.

The application of lossy compression schemes in a networked teleoperation system reduces packet transmission rates making real- 
time teleoperation amenable. But it also introduces distortions into the haptic feedback signals and therefore deterioration of signal quality. Given a teleoperation system, we seek to optimize compression scheme parameters, so as to have good quality, while maintaining low packet rates over the communication channel.

This paper is organized as follows. In Section 2, we describe the haptic data reduction scheme that shall be evaluated for quality in this work. Section 3 presents the proposed objective quality evaluation framework for haptic compression schemes. Further, under this framework, Section 4 divides the human operator model into two parts - the model of the human control action and that of the haptic perception process, and describes experiments for identifying model parameters. Section 5 discusses model simulation results and the paper is concluded with suggestions for future refinements to the models and the framework in general.

\section{HAPTIC DATA REDUCTION}

Transmitting haptic signals over packet-switched networks is characterized by strict delay constraints as well as by high data and packet rates. Our previous work has shown that these challenges can be successfully addressed by the so-called deadband approach [7]. It allows us to significantly reduce the amount of haptic data as well as the network packet rate without impairing the system's quality as shown by human user studies [7]. By exploiting Weber's law of Just Noticeable Differences (JND), it allows us to remove haptic information that is imperceptible by the human. In the following, its background, principle and application are explained.

\subsection{Weber's law of Just Noticeable Differences}

Psychophysical studies have revealed that haptic perception of forces, velocity, pressure, etc. follows a mathematical relationship between the physical intensity of a stimulus and its phenomenologically perceived intensity [19]. In 1834, Ernst Weber proposed the size of the difference threshold to be a linear function of stimulus intensity. This has become known as Weber's law of JNDs:

$$
\frac{\Delta I}{I}=k \quad \text { or } \quad \Delta I=k \cdot I,
$$

where $I$ is the initial stimulus and $\Delta I$ is the so called Difference Threshold (or the JND). It describes the smallest amount of change of stimulus $I$ which can be detected as often as it cannot be. The constant $k$ (called the deadband parameter $k$ from now on) describes the linear relationship between $\Delta I$ and the initial stimulus $I$.

\subsection{Haptic data reduction using perceptual deadbands}

Based on Weber's Law, insignificant changes in the processed force feedback data stream are deemed undetectable and corresponding haptic samples can be dropped. Only if the difference between the most recently sent sample and the current value exceeds human perception thresholds, a new signal update is triggered. At the receiver, a simple zero-order hold strategy allows for upsampling the irregularly received signal updates to a constant and high sampling rate required for feeding the local control loops.

The principle of deadband-based data reduction is illustrated in Fig. 1 for the force feedback channel. Samples shown as filled circles represent the output of the deadband coding scheme. They define perception thresholds represented by a perceptual deadband, illustrated as gray zones. Using the insights provided by Weber's

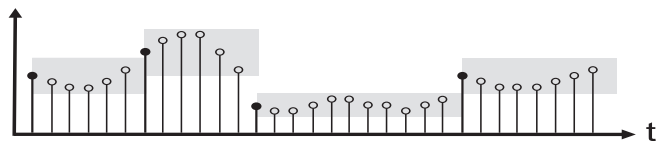

Figure 1: Principle of deadband-based data reduction.

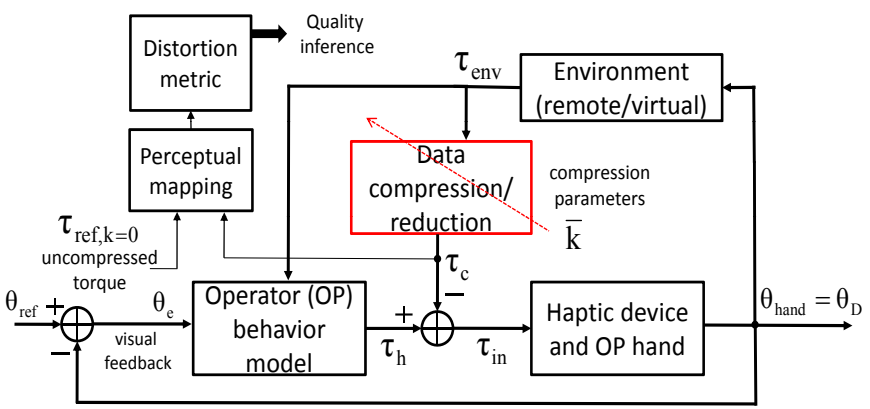

Figure 2: Block diagram of the OQE framework for haptic data compression.

law, the applied deadband size grows in direct proportion to the magnitude of the most recently transmitted and applied haptic sample, the constant of proportionality being $k$. For haptic perception, $k$ has been found to be in the range of $5 \%$ to $15 \%$, depending on the type of stimulus and the limb/joint where it is applied [2]. If the haptic signal exceeds expected human perception thresholds then a signal update is triggered to be sent over the network, which also redefines the applied deadband threshold.

In the following, the phrases 'haptic compression' and 'haptic data reduction' are used interchangeably. They both refer to schemes aimed at reducing network traffic by minimizing intersample transmissions as described above rather than the size of intrasample content.

\section{A QUALITY EVALUATION FRAMEWORK FOR HAPTIC DATA REDUCTION}

While analyzing performance of different compression schemes or different settings of the same scheme, fairness demands having a common basis for comparison. This is automatically ensured in audio and video coding schemes since exactly the same media content can be subjected to compression and tested for in subjective as well as objective evaluation. The additional manipulative ability afforded in haptics, however, might result in possibly different user experiences, on account of performed actions among users deviating from each other. It follows that there is a need for standardization of description of the task to be performed by the user, to enforce similar experiences as far as is possible. This motivates us to consider a trajectory tracking task, for which operator models have been derived in detail in the past $[6,16]$. A specified position signal for such a task will standardize measurements across all users, for a given static environment.

It is important to note here that the choice of task and the human control model that is elicited from it has no influence on the efficacy of the results. The results are applicable at a signal-level, independent of the varied descriptions of haptic interaction tasks in practice. Our sole purpose of investigating a tracking task is to buy a definite degree of control over physical stimuli that different subjects will experience, by regulating the user input to the system over a reasonably wide dynamic range in amplitude and frequency. This range will be dictated by device limits and limits of human manipulation.

In our setting, the user performs a compensatory tracking task. Tracking error is visually displayed and the user attempts to keep this error at a minimum while being subjected to force feedback generated from the environment. This is a kind of negative feedback control, where the user applies corrections through the physical motion of his muscular system to achieve a specified control objective. Figure 2 shows an abstract block diagram of the proposed framework.

The point-to-point error between the reference trajectory to be followed $\theta_{\text {ref }}$ and the actual trajectory $\theta_{o}$ is fed back to the operator visually. The operator responds to this error by producing a torque $\tau_{h}$ while continuously perceiving the torque reflected by 
the haptic device. Together with $\tau_{h}$, the torque $\tau_{\text {env }}$ generated by the environment in response to user motion collectively drives the hand-device model to produce a position signal at its output. The output position of the device on the operator (OP) side is communicated to the remote environment over the forward communication channel. In the other direction, before the environment torque $\tau_{e n v}$ is sent over the reverse channel to the OP side for display, the data compression scheme operates on it to minimize transmission rate. Altering the compression scheme parameter vector $\vec{k}$ appropriately allows us to tune the strength of compression. For lossy compression schemes, it generally holds that the stronger the compression, the larger the savings in the transmission rate, but larger the distortion introduced into the feedback signal. For a given parameter setting, the compressed feedback signal $\tau_{c}$ is mapped to the perceptual domain along with the reference uncompressed one $\left(\tau_{r e f, k=0}\right.$, where $k=0$ implies that the deadband scheme is inactive) based on psychophysical laws. A distance metric measures the distortion of the compressed signal in the perceptual domain with respect to the uncompressed one, inverting it to produce an estimate of the feedback signal quality. We construct a linear model of the dynamics of such an haptic interaction in the following.

\section{Modeling the hUMAN OPERATOR}

In the following, we divide the framework into two main parts - 1 . model of the active control behavior of the human operator, and 2. model of the human haptic perceptual mechanism. Such a partition of the human model is a simplification that is necessary to make the modeling problem tractable.

\subsection{The human operator as a controller}

A well established isomorphic structural model of the human operator for compensatory manual control is presented in [6] and is shown here in Figure 4 (dashed box). It was applied by Penin et $a l$. for simulating a teleoperation system with kinesthetic feedback in [16]. The internal feedback structure of this model reflects the hypothesized human proprioceptive feedback activity. The model has been divided into the central nervous system and the neuromuscular system that clearly delineates the signal processing involved. Control signals $u_{c}(t)$ are produced by the human, proportional to the tracking error (the proportionality constant being $K_{e}$ ) and inherently delayed $\left(d_{o}\right)$ by transport along neuro-motor signal pathways and response dynamics (e.g. visual stimulus processing delay). The control signal $u_{c}(t)$ is the input command to the closed-loop system consisting of the active open-loop neuromuscular dynamics $Y_{p_{n}}$ of the arm and elements $Y_{f}$ and $Y_{m}$. These elements represent in an approximate fashion the combined effects of the muscle spindles, Golgi tendon organs, joint angle receptors and the dynamics associated with higher level signal processing. As proposed by Hess in [6], for the neuromuscular system, the following characteristic values were selected: $\omega_{n}=10$ and $\zeta=0.707$.

In the tracking task, the hand operates in conjunction with the haptic device manipulator. The passive hand and device characteristics are represented by the block diagram shown in Figure 3. The superposition of the device actuator torque and the torque commanded by the CNS is denoted by $\tau_{\text {in }}$. Output $\theta_{\text {hand }}=\theta_{D}$ denotes the collective position of the hand and the device. The human hand impedance is modeled as a linear time-invariant 2 nd order model with effective mass $M_{h}$, damping $B_{h}$ and stiffness $K_{h}$ :

$$
H(s)=\frac{\tau_{\text {hand }}(s)}{\theta_{\text {hand }}(s)}=M_{h} s^{2}+B_{h} s+K_{h}
$$

The transfer function of the haptic device dynamics with mass $M_{d}$ and damping $B_{d}$ can be represented as:

$$
D(s)=\frac{\theta_{D}(s)}{\tau_{D}(s)}=\frac{1}{M_{d} s^{2}+B_{d} s}
$$

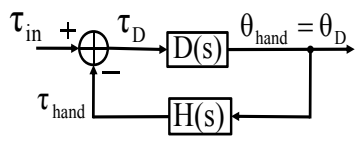

Figure 3: hand-device model structure.

From (2) and (3), we have the overall transfer function:

$$
\begin{aligned}
H D(s) & =\frac{\theta_{D}(s)}{\tau_{i n}(s)}=\frac{D(s)}{1+D(s) \cdot H(s)} \\
& =\frac{1}{\left(M_{h}+M_{d}\right) s^{2}+\left(B_{h}+B_{d}\right) s+K_{h}}
\end{aligned}
$$

where $M \triangleq\left(M_{h}+M_{d}\right), B \triangleq\left(B_{h}+B_{d}\right)$ and $K \triangleq K_{h}$ further on.

The hand-device model fits into the rest of the operator control framework which we refer to later on as the operator model, as shown in Figure 4. Again, under the assumption of linearity, the operator output torque $\tau_{h}$ together with the torque $\tau_{\text {env }}$ generated by the environment ( $\tau_{c}$ after compression) drive the hand-device model.

For this work, we neither insist on nor claim rigor. Human operator modeling has been studied in great depth and much more detailed models for tracking tasks have been developed [15]. Recently, researchers have also begun to focus on tracking tasks with haptic feedback [12]. Our intention here is primarily to lay the foundations of a preliminary framework which would be subject to refinements in the future.

\subsubsection{Parameter estimation}

Parameters are estimated for the system in two successive experiments. In the first experiment, we identify the hand-device model for a specified grip force typical of desktop haptics. Thus dependence of the passive hand dynamics on the grip force can be ignored. The parameter values determined from the first experiment are then fixed in the second experiment, where we identify the operator model that drives the hand-device model.

Experimental setup Experiments were performed using a PHANToM Omni haptic device (Sensable Technologies Corp., Woburn, MA) equipped with a FlexiForce force sensor to sense grip forces (TekScan Corp., Boston, MA). We made use of only the first (base) joint of the haptic device for simplicity. A usb sensor interface kit from Phidgets Inc. (Calgary, Alberta, Canada) acquired data from the grip force sensor. A real-time executable application built using MATLAB/Simulink running on a standard Windows PC (dual core, $2.13 \mathrm{GHz}$ ) ran a servo loop that reads the high-precision Omni device position encoders and commanded torque values to it at the standard $1 \mathrm{kHz}$ haptic sampling rate.

Subjects Nine right hand dominant subjects ( 7 male, 2 female, ages 20-30 yrs.) participated in this study. All subjects were healthy with fully operational limbs and tested using their (dominant) right arm. The same subjects participated in both the experiments. The experimental procedures were reviewed in detail before each experiment and are described below.

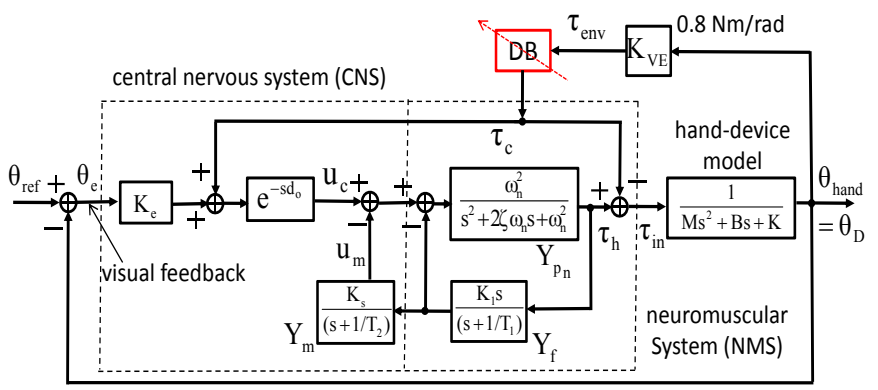

Figure 4: Structural model for the compensatory tracking task. 


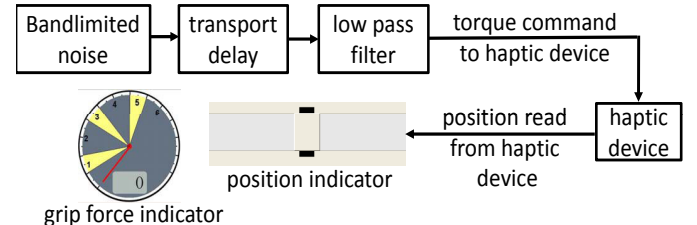

Figure 5: Experiment 1 - identification of the hand-device model. A transport delay is added to allow the subject a time duration of 1 second to position the slider exactly at the center before the experiment began.

Experimental procedure In the hand-device identification experiment (see Figure 5), the subjects were displayed a slider on the screen which corresponded directly to the position of their hand (the same as that of the device). Standard system identification methods using input signals such as frequency sweeps, discrete sinusoidal signals, and random noise produce comparable results. However, we commanded a Gaussian white noise force input to the device, low pass filtered with a cutoff frequency of 30 $\mathrm{Hz}$ [4]. This is because when the human hand is subjected to random force disturbances, it is less likely to trigger its reflexes (which our model does not account for). The subjects were instructed to attempt to hold the visual slider position at the center without forcing the device against the applied torque, while maintaining a grip force of roughly around $3 \mathrm{~N}$ typical to desktop haptic applications. A visual cue in the form of a dial was shown on the screen too, so that the subjects could correct their grip force when necessary. The commanded input torque and the corresponding output position of the haptic device were recorded for subsequent analysis. Figure 7 shows an excerpt of the time domain input and output signals recorded during the experiment. The low pass character of the hand-device combination is evident herein.

In the operator model experiment (see Figure 6), subjects were displayed the tracking error with respect to the reference trajectory. They were then asked to attempt to keep the error at a minimum by adjusting hand position to make the slider position approach the center of the scale, which corresponds to zero tracking error. They were well trained for this tracking task before the actual experiment. Proportional force feedback according to a spring model (with stiffness $K_{V E}=0.8 \mathrm{Nm} / \mathrm{rad}$ ) for the environment was computed and commanded to the haptic device to be applied to the hand after undergoing deadband compression. The subjects were requested to maintain a consistent grip force on the device, for which purpose a visual cue is provided, similar to the first experiment. The reference input trajectory, the actual trajectory traced by the user and the corresponding feedback torque were recorded. Figure 8 shows an example of the time domain input signal that was to be followed and the corresponding actual signal that the user produced in response.

Each subject was initially trained to recognize the step-like deadband artifacts haptically. He then performed the tracking task (100 sec. long) multiple times for a range of deadband parameter $k$ values. To avoid learning effects, the $k$ values were chosen randomly across experimental runs. After each run, a subjective rating corresponding to the perceived distortion in the haptic feedback because

Table 1: Hand-device model parameters (rounded up for convenience)

\begin{tabular}{c|c|c}
$M\left(\mathrm{kgm}^{2}\right)$ & $B\left(\mathrm{kgm}^{2} / \mathrm{rad}\right)$ & $K(\mathrm{Nm} / \mathrm{rad})$ \\
\hline $0.003 \pm 0.002$ & $0.45 \pm 0.21$ & $9.25 \pm 3$
\end{tabular}

Table 2: Operator structural model parameters (rounded up for convenience)

\begin{tabular}{c|c|c|c|c}
$K_{e}$ & $d_{o}(\mathrm{sec})$ & $K_{1}$ & $K_{2}$ & $\frac{1}{T_{1}}, \frac{1}{T_{2}}\left(\mathrm{sec}^{-1}\right)$ \\
\hline $141 \pm 34$ & $0.28 \pm 0.07$ & $65 \pm 15$ & $38 \pm 10$ & $23 \pm 7$
\end{tabular}

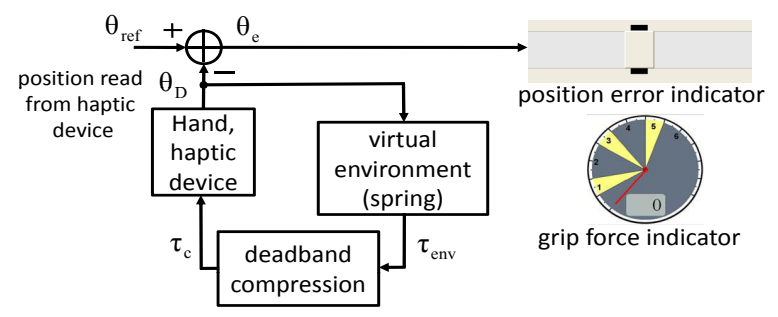

Figure 6: Experiment 2 - human model identification.

of the compression scheme was solicited and recorded (see [7] for a more detailed description of the rating scale). It is noted that for a reasonable range of $\mathrm{k}$, no operational instabilities have been observed during experiments even though no additional measures for the passification of the deadband scheme have been implemented (see [9] for details). This may be attributed to device and human damping, which dissipate energy injected into the haptic loop.

System identification Figure 9 shows the Bode plot of the transfer function estimated from the first half of input-output data measured in the hand-device identification experiment, averaged across all users. Averaging was possible since exactly the same input torque was used for each of the users. A second order transfer function was then fitted to the experimental data, and the estimated parameters are listed in Table 1. The second half of the experimental data was used for model validation.

For the second experiment, the input signal was designed to be a summation of thirteen sinusoids to ensure a random appearing input to the user. The input frequencies were distributed evenly over the range interesting for manual control $\left(0 \leq f_{\text {in }} \leq 3 \mathrm{~Hz}\right)$ [6]. The presence of delay $d_{o}$ in the OP model makes parameter adjustment in the frequency domain difficult and therefore parameter values (Table 2) were estimated in the time domain by least squares-based optimization. An initial guess of $d_{o}=0.2 \mathrm{sec}$ was used for the OP delay [6].

\subsection{Haptic perceptual mapping}

In this section, we present a haptic perception model based on a well established law of psychophysics, namely the Weber-Fechner law [20]. Inspired by this law, we hypothesize that the psychological sensation registered by the brain when experiencing haptic stimuli varies logarithmically with the magnitude of the physical haptic stimulus:

$$
S=c \cdot \ln \left(\frac{x}{x_{o}}\right)
$$

where $S$ denotes the perceived sensation, $c$ is a scaling constant typically determined experimentally, $x$ represents the stimulus magnitude, and $x_{o}$ denotes the absolute threshold below which no stimulus can be perceived at all.

For an $N$-long time-sampled signal, we define a distortion metric -perceptual mean square error or pmse - in the perceptual domain as:

$$
\begin{aligned}
\text { pmse } & =\frac{1}{N} \sum_{i=0}^{N-1}(S(i)-\hat{S}(i))^{2} \\
& =\frac{c^{2}}{N} \cdot \sum_{i=0}^{N-1}\left(\ln \left(\frac{x(i)}{\hat{x}(i)}\right)\right)^{2}
\end{aligned}
$$

where in the perceptual domain, $S$ is the undistorted reference, while $\hat{S}$ is the signal distorted by data compression, $x$ and $\hat{x}$ being the corresponding time-domain signals. 

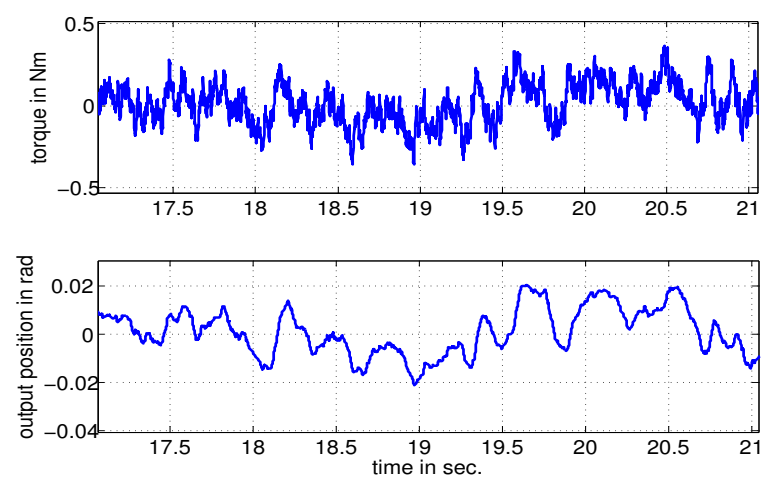

Figure 7: Experiment 1 - time domain plots.

\section{Simulation RESULTS}

With the model parameters obtained before, the model of the human operator interacting haptically with an environment is simulated for a range of values of the compression scheme parameter $k$. The quality of the resultant compressed force feedback signals, distorted due to the lossy compression scheme is evaluated using our metric pmse for perceptual distortion. Figures 11, 12, 13 and 14 show the time domain position and feedback torque waveforms for a couple of deadband parameter $k$ values. Figure 15 shows results of the simulations performed for various values of the deadband parameter $k$. It can be seen that with increasing $k$, the predicted perceived signal quality as well as transmission packet rates decrease.

Figure 16 shows subjective quality ratings obtained experimentally (experiment 2) for comparison. It can be seen here that the trend of the quality curve plotted against increasing values of the deadband parameter $k$ is coherent with that predicted by the simulations.

\section{CONCLUSIONS AND FUTURE WORK}

A framework and a methodology for objective quality evaluation of haptic compression schemes was proposed and validated by experiments and simulations. Quality results predicted by the simulations match in trend with those obtained from psychophysical tests. The optimal compression parameter $k$ can be chosen from the simulation results depending upon what operation quality is regarded as acceptable. By design, the haptic task investigated in this work generates low-frequency haptic feedback. The perceptual distortion metric deployed is especially well suited to capture artifacts within this framework. However, the detection of more complex features (e.g. communication artifacts arising out of receiver-side signal reconstruction in presence of packet loss, network delay and jitter) in the haptic signals will necessitate more complex psychophysical models.

More detailed models of the human manual control action exist that, for instance, additionally consider vestibular feedback, an

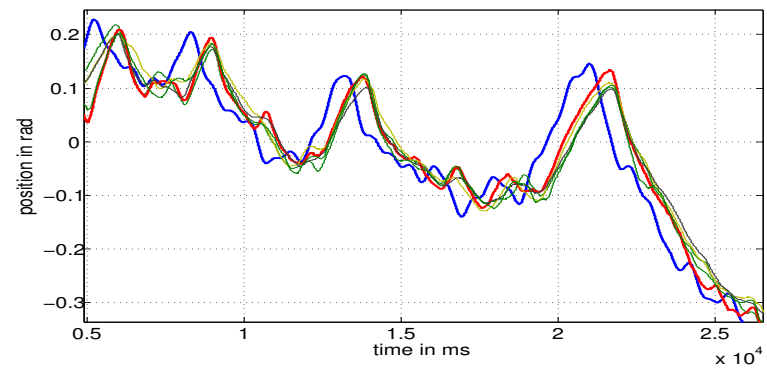

Figure 8: Experiment 2 - time domain plots: blue curve shows reference position signal to be followed, all other curves are the actual traced position signals for a range of deadband $k$ values, averaged across users. Variances in model parameters across the $k$-range are shown in Table 2 .
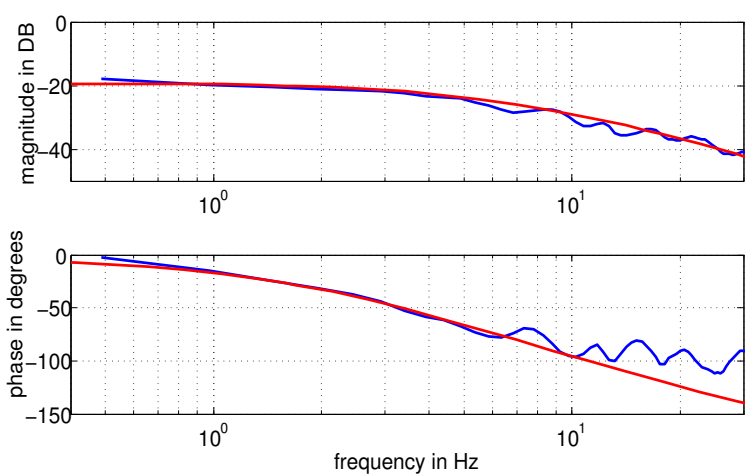

Figure 9: Experiment 1 - Bode plots. The red curve represents the transfer function fitted to the experimental one shown in blue.

important component of the human movement regulating and stabilizing system. Remnant signals that account for the non-linearities that are not captured by the linear human model have also been modeled in detail by researchers. The adaptability of such models for our purpose needs to be evaluated in the future. Moreover, a comprehensive model of the human haptic perception needs to be developed. It is expected that such future refinements shall improve the statistical correlations between objective quality results obtained by simulation and those from subjective tests.

\section{ACKNOWLEDGEMENTS}

The authors would like to thank the German Research Foundation (DFG) for supporting this work under project STE 1093/4-1.

\section{REFERENCES}

[1] C. Basdogan, C.-H. Ho, M. A. Srinivasan, and M. Slater. An experimental study on the role of touch in shared virtual environments. $A C M$ Transactions on Computer-Human Interaction, 7(4):443-460, 2000.

[2] G. C. Burdea. Force and touch feedback for virtual reality. John Wiley \& Sons, Inc., New York, NY, USA, 1996.

[3] J. Colgate, J.E.and Brown. Factors affecting the z-width of a haptic display. Robotics and Automation, 1994. Proceedings., 1994 IEEE International Conference on, 4:3205-3210, May 1994.

[4] M. Fu and M. Cavusoglu. Three-dimensional human arm and hand dynamics and variability model for a stylus-based haptic interface. Robotics and Automation (ICRA), 2010 IEEE International Conference on, pages 1339-1346, 2010.

[5] A. Hamam, M. Eid, A. Saddik, and N. D. Georganas. A fuzzy logic system for evaluating quality of experience of haptic-based applications. In Proceedings of the 6th international conference on Haptics: Perception, Devices and Scenarios, EuroHaptics '08, pages 129-138, Berlin, Heidelberg, 2008. Springer-Verlag.

[6] R. A. Hess. Structural model of the adaptive human pilot. Journal of Guidance and Control, 3(5):416-423, 1980.

[7] P. Hinterseer, S. Hirche, S. Chaudhuri, E. Steinbach, and M. Buss. Perception-based data reduction and transmission of haptic data in
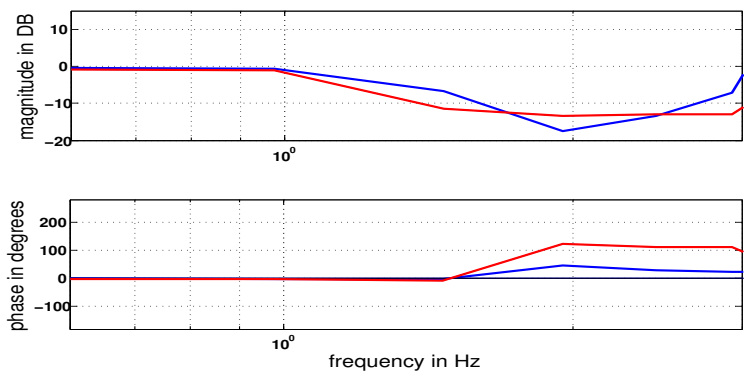

Figure 10: Experiment 2 - Bode plots. The red curve shows the transfer function fitted to the experimental one shown in blue. 
telepresence and teleaction systems. IEEE Transactions on Signal Processing, 56(2):588-597, Feb. 2008.

[8] S. Hirche and M. Buss. Transparenz haptischer telepräsenzsysteme mit konstanter zeitverzögerung (transparency of haptic telepresence systems with constant time delay). Automatisierungstechnik, 54(2):51-59, 2006.

[9] S. Hirche, P. Hinterseer, E. Steinbach, E. Steinbach, and M. Buss. Network traffic reduction in haptic telepresence systems by deadband control. In In: Proceedings of the 16th IFAC World, 2005.

[10] P. Hokayem and M. Spong. Bilateral teleoperation: An historical survey. Automatica, 42(12):2035-2057, 2006.

[11] U. Jekosch. Voice and Speech Quality Perception. Springer Verlag, 2005.

[12] T. Lam, M. Mulder, and M. van Paassen. Haptic interface in uav tele-operation using force-stiffness feedback. Systems, Man and $C y$ bernetics, 2009. SMC 2009. IEEE International Conference on, pages 835-840, 2009.

[13] D. Lawrence. Stability and transparency in bilateral teleoperation. Proc. of the 31st IEEE Conference on Decision and Control, pages 2649-2655, 1992.

[14] D. Lawrence, L. Pao, A. Dougherty, M. Salada, and Y. Pavlou. Ratehardness: a new performance metric for haptic interfaces. Robotics and Automation, IEEE Transactions on, 16(4):357-371, Aug 2000.

[15] D. McRuer. Human Dynamics in Man-Machine Systems, volume 16. Automatica, 1980.

[16] L. Penin, A. Caballero, R. Aracil, and A. Barrientos. Human behavior modeling in master-slave teleoperation with kinesthetic feedback. Robotics and Automation, 1998. Proceedings. 1998 IEEE International Conference on, 3:2244-2249, 1998.

[17] M. Sargardia, T. Hulin, C. Preusche, and G. Hirzinger. A benchmark of force quality in haptic rendering. Proceedings of the 13th International Conference on Human-Computer Interaction (HCI), 2009.

[18] Z. Wang, H. R. Sheikh, and A. C. Bovik. Objective video quality assessment. In B. Furht and O. Marqure, editors, The Handbook of Video Databases: Design and Applications, chapter 41, pages 10411078. CRC Press, September 2003.

[19] E. Weber. Die Lehre vom Tastsinn und Gemeingefuehl, auf Versuche gegruendet. Vieweg: Braunschweig, Germany, 1851.

[20] H. Yilmaz. On the laws of psychophysics. Bulletin of Mathematical Biology, 26:235-237, 1964. 10.1007/BF02479044.

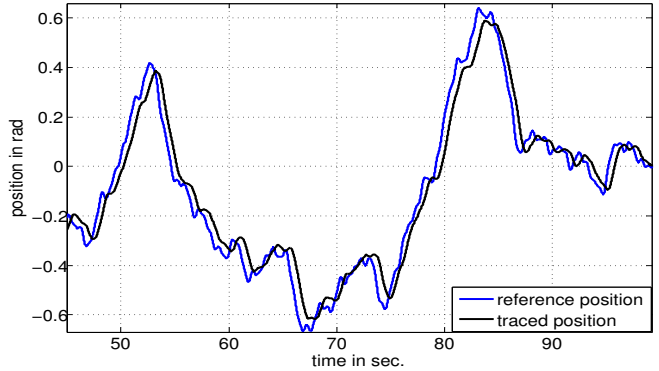

Figure 11: Simulation waveforms for deadband parameter $k=0$ : positions.

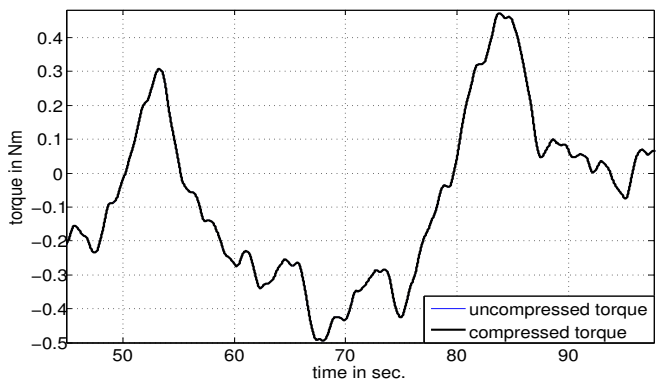

Figure 12: Simulation waveforms for deadband parameter $k=0$ : torques (the uncompressed and compressed waveforms coincide for $k=0$ ).

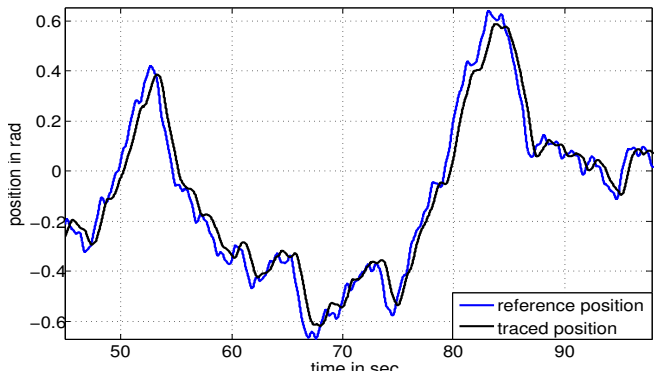

Figure 13: Simulation waveforms for deadband parameter $k=0.15$ : positions.

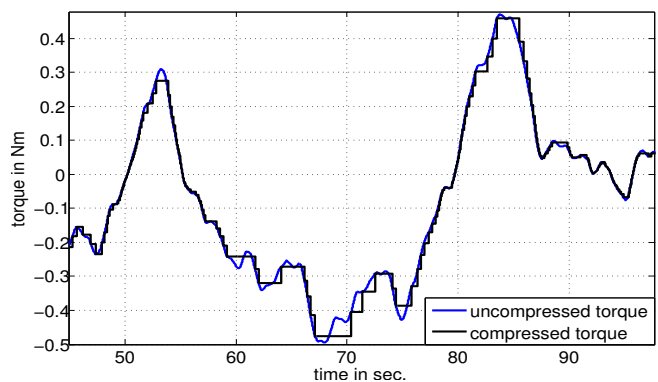

Figure 14: Simulation waveforms for deadband parameter $k=0.15$ : torques. Flat areas on the black curve represent areas of no packet transmission. Each vertical step on this curve represents one packet transmission.
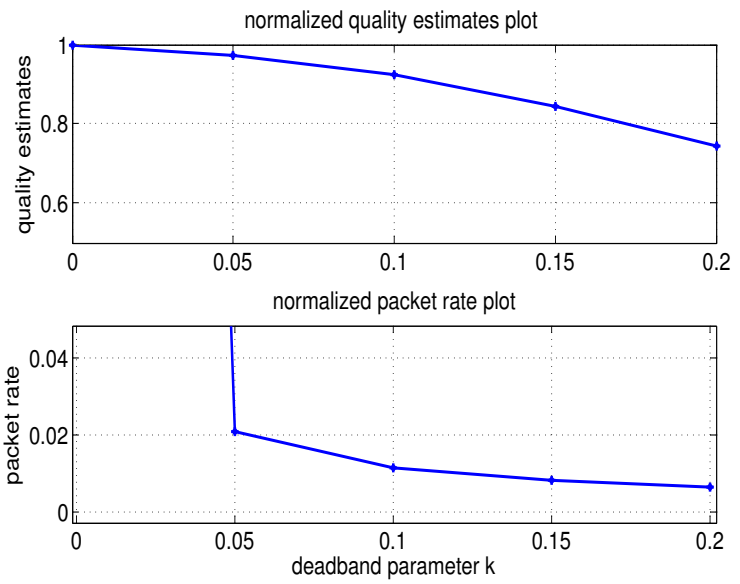

Figure 15: Quality estimates using pmse and packet rate simulation results. The normalized packet rate at $k=0$ is 1 .

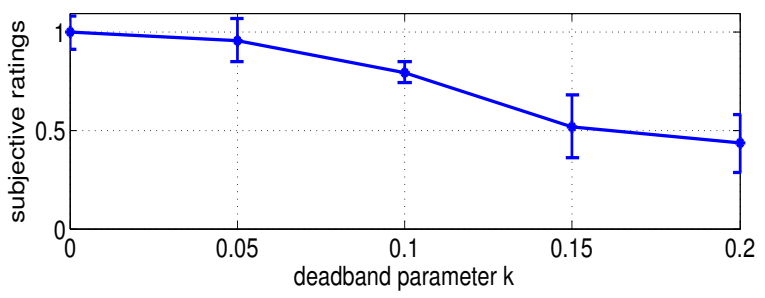

Figure 16: Normalized mean subjective quality ratings obtained from experiment 2 , with corresponding standard deviations. 